\title{
61.3: Invited Paper: A Virtual Display for Mobile Use
}

\author{
Jukka Häkkinen \\ Audio-Visual Systems Laboratory, Nokia Research Center, Helsinki, Finland
}

\begin{abstract}
Mobile devices have small displays, so a virtual display that would bring the benefits of a large computer display to users would be an interesting mobile accessory. However, sickness symptoms are a problem that should be solved before a virtual display can be successful. To explore the symptom levels induced by virtual displays, we have tested several head-worn virtual display types in various contexts. Our results indicate that monocular and stereoscopic displays induce significant amount of adverse symptoms. On the other hand, the symptom levels induced by biocular displays are not different from the symptoms induced by direct view displays. The results suggest that a biocular display might be the best alternative as a mobile accessory.
\end{abstract}

\section{Introduction}

A head-worn virtual display would be an excellent mobile accessory because it would bring the benefits of a large computer display to a mobile device. Navigating the web or using office applications would be comparable to laptop computer work, and watching a movie or playing a game could be a more exciting and immersive experience than with a tabletop display.

However, many have heard about the sickness symptoms caused by virtual displays. According to the stories and experimental results, people become nauseous and get headaches after using such a display. $[1,2,3,4]$. For example, in a large study completed by Cobb and al [1] $80 \%$ of the users experienced some level of symptoms. With $5 \%$ of the users the symptoms were so significant that they had to interrupt the use before the end of the test. The subjective symptoms are sometimes accompanied by disruptions in performance. For example perception, motor performance and postural stability can be disrupted [1]. The subjective symptoms affect the enjoyableness of the use, but degraded performance can also be a safety issue, because the aftereffects can affect the user's ability to walk, drive a car, use machinery or perform other sensomotorically demanding tasks. Furthermore, in worst case such effects can persist for hours after the immersion [2].

The possibility of virtual display induced symptoms and effects has led some researchers to considerable skepticism about the viability of consumer virtual displays. To study these effects, we have been conducting large virtual displays studies over the last few years in the Nokia Research Center. So far, we have conducted 17 experiments and 440 users have participated in them and we are beginning to form a general view about the key ergonomics issues in virtual displays.

In our tests we have used monocular, biocular and binocular stereoscopic displays in various tasks and contexts. Our test equipment has included for example Sony Glasstron, Olympus EyeTrek, Olympus PCEyeTrek, Xybernaut Poma and MicroOptical BV-3 head-worn displays. In this article I will report some technology related findings of our research program.

\section{Methods}

The data gathered during the experiment can be categorized to the following categories: 1) Background information collection, 2) Cybersickness and visual strain questionnaires 3) Visual system measurements, and 4) Aftereffect measurements.

\subsection{Background information}

Background information was collected with a questionnaire whose purpose was to concentrate to issues that might be relevant in head-worn display context. Firstly, the participants were asked about experiences that might affect their performance and experiences during use. Specifically, they were asked to write a general work description, and report any previous head-worn display experience. Also, they were asked to estimate the time spent watching television or computer monitor, and the time spent playing computer games. The rationale behind these questions was to find out whether participants with previous experience with head-worn displays and gaming react differently to the usage situation. Secondly, subjects were asked about to rate their susceptibility to headache and motion sickness.

\subsection{Cybersickness}

Cybersickness is a general feeling of discomfort and is often associated with nausea and stomach symptoms. Symptoms include nausea, burping, vomiting, retching, pallor, sweating, bodily warmth, salivation, anxiety, apathy, headache, sensation of heavy eyelids, "fullness of the head", drowsiness, hyperaesthesia of the scalp, ocular pain associated with extraocular and intraocular muscle, vertigo, dyspepsia, stomach awareness, disorientation, postural instability and residual aftereffects [6,7]. The symptoms are similar to motion sickness and seasickness and can be characterized as symptoms that occur when people are exposed to stimuli they are unfamiliar and unadapted with.

In this experiment we used Simulator Sickness Questionnaire to quantify the amount of subjective symptoms. The SSQ produces four scores that characterize the user experience with the headworn display. The total score indicates the general symptom level and three symptom factors (nausea, oculomotor and disorientation) give more specific information. Because individual differences in the rating scales and strategies are large, usually both pre- and post-immersion ratings are obtained so that the individual variation can be eliminated. Thus, the resulting score represents the differences between pre- and post-immersion ratings. Visual Symptoms Questionnaire (VSQ) was used to quantify the feelings of visual strain. 


\subsection{Visual screening}

Visual status of the participants was screened before each experiment. These tests included near and far visual acuity, stereo acuity, interpupillary distance, fixation disparity, eye dominance and convergence ability, $\mathrm{AC} / \mathrm{A}$ ratio.

\subsection{Dynamic visual measures}

It can be assumed that the visual system is somehow affected during the head-worn display use, so the functioning of the system should somehow be quantified before and after using a virtual display. In our studies we measured the functioning of the accommodation system and latent deviation of the visual axes, heterophoria.

\subsection{Aftereffects measurement}

In some experiments the postural sway of the participants was measured with AccuSway postural platform. Participants stood in the platform for 30 seconds with eyes open and for 30 seconds with eyes closed. The speed of the center-of-pressure (COP) was used as a measure for the postural sway. The participants' sway was measured before the task and it was compared to post-task measurements that were started immediately after the task and were continued in 10-minute intervals for 60 minutes.

\section{Results}

The participants of the experiments were from Nokia Research Center and University of Helsinki (The general background variables are described in Table 1). Their mean age was 29 years and they were predominantly male. Most of them wore glasses and did not have previous experience of head-worn displays.

\subsection{Monocular displays}

The main ergonomic problem with monocular displays is binocular rivalry, which is caused by the different views perceived by the left and right eyes. During rivalry the dissimilar views from the left and right eye are seen alternately rather than as a single visual world. For the user binocular rivalry is an annoying and straining experience. Objective tests also demonstrate that in a

\begin{tabular}{|c|c|}
\hline Background variable & \\
\hline Mean age & 29.23 years \\
\hline Gender & $\begin{array}{c}\text { Males } 66 \% \\
\text { Females } 34 \%\end{array}$ \\
\hline Glasses? & $\begin{array}{l}\text { Yes } 60 \% \\
\text { No } 40 \%\end{array}$ \\
\hline $\begin{array}{l}\text { Previous experience with head-worn } \\
\text { displays? }\end{array}$ & $\begin{array}{l}\text { Yes } 39 \% \\
\text { No } 61 \%\end{array}$ \\
\hline $\begin{array}{c}\text { Average television watching/ computer } \\
\text { working time / day }\end{array}$ & $7.23 \mathrm{~h}$ \\
\hline
\end{tabular}

Table 1. Experiment participants rivalry situation the muscles, which control the focusing system of the eyes and the movement of the eyes, get strained. Users can learn to control the rivalry with training, and monocular displays are used in various military and technical contexts. However, it is questionable whether a consumer product user would be willing to go through such a training period.

Figure 1 shows the subjective symptom levels that from an experiment that compares monocular display (Xybernaut Poma) and biocular, below the eye display (MicroOptical BV-3). In the test series the users were required to complete a task in which they had to glance between the display and the surroundings. The participants read a text from a paper and compared it to a text, which was shown in a virtual display. When they noticed any differences, they edited the text that was shown in the virtual display. The task duration was 40 minutes. To compare different display types, the same task was performed with standard CRT monitor, MicroOptical BV-3 biocular head-worn display and with Xybernaut Poma monocular display. 20 users participated in each experimental group, so a total of 60 users participated the experiment. The figure clearly shows that one-eye display produces significantly more symptoms than the biocular display or a CRT. User interviews indicated that some users experienced adverse symptoms several hours after the monocular display use.

Although one-eye displays have many ergonomics problems, there are use cases in which they might be useful. A monocular display could be usable as a short-term display for usage situations that last 5 to 10 minutes. However, special attention should be given to the positioning of the virtual display, and the user should be able to put on the display within seconds and without conscious effort. The importance of a good fit of a display is shown in our test of Olympus PC EyeTrek monocular display, in which we recorded the number of times that the users modified the position of the display during a 40 minute experiment. The results show that $43 \%$ of subjects modified the display over four times during the experiment (at least once in every 10 minutes) and $8 \%$ of subjects had to modify the head-worn display over 15 times (at least once in every 2.5 minutes). The number of required modifications correlated directly to the symptom levels and general use comfort.

Our test results apply only to monocular displays that cover the whole visual field in one eye. Smaller displays that cover the visual field only partially may be more comfortable alternative, because binocular rivalry is decreased.

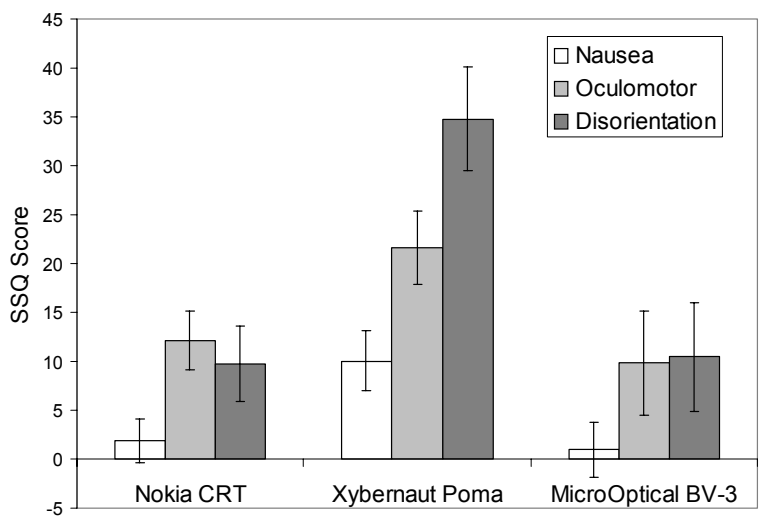

Figure 1. Symptom levels in three different experimental conditions. 


\subsection{Stereoscopic displays}

Binocular displays do not have the problems with binocular rivalry or other issues that constrain the use of one-eye displays. A stereo image can be a fascinating experience, so a binocular stereoscopic display can be seen as a tempting alternative as a mobile accessory. However, a stereo image causes conflicting demands for the muscles that control the movement of the eyes and the focusing of the lens system of the eye. This convergenceaccommodation conflict is the main reason for eyestrain with stereoscopic virtual displays. Although there might be technological and UI related solutions to the problem, this might still delay the introduction of a stereoscopic head-worn display. The significance of these effects was demonstrated in our experiments, in which stereoscopic displays caused significantly more symptoms than non-stereoscopic displays.

\subsection{Biocular displays}

A biocular display has two displays but the same image is shown to both eyes. This solution avoids the problems that are associated with monocular displays and with stereoscopic displays. Most importantly, because the images are the same, the convergence and accommodation stay in the same plane and eyestrain is minimized. Our tests indicate that a biocular display is the most comfortable alternative as a mobile virtual display. In the following sections I will describe two such experimental results.

\subsubsection{Gaming and movie watching}

In one experimental series we compared the user experiences with three different tasks. One group watched a movie from a television for 40 minutes. The second group used an Olympus EyeTrek head-worn display in a biocular mode to watch the same movie. And the third group played a car racing game with the same Olympus glasses. Each group consisted of 20 participants.

We measured subjective, ophthalmological, and physiological variables, but here I will concentrate on the subjective experiences. The subjective symptoms were measured with the Simulator Sickness Questionnaire (SSQ) [5]. The users completed the questionnaire before and after the task in 10-minute intervals for 60 minutes. The resulting score represents the difference in subjective symptom levels before and after the task.
Figure 2 shows the symptom levels after the users performed the task. The subjective symptoms are divided into three factors, each of which correlates to specific neural systems: nausea, oculomotor, and disorientation symptoms. The curves demonstrate several important results. Firstly, gaming (triangle markers) produced a lot of symptoms: all the symptom levels are elevated 10-30 minutes after the task. This increase in subjective symptoms probably correlates to disruptions in sensomotoric performance.

As could be expected, watching a movie from a television does not cause a lot of symptoms (circle markers). Interestingly, when the users watched a movie with the biocular display (square markers), the symptom levels were the same as with the television with the exception of nausea symptoms (the leftmost curves in Figure 1). The nausea subjective symptom levels had negative values after the task, which means that the users felt better after using the virtual display than before. We relate this result to the fact that the users came to the experiment during a busy working day and may have been slightly stressed. The result shows that a biocular virtual display reduced the stress symptoms more than watching the same movie from a television. This is a good indication that a biocular display provides a good and comfortable user experience.

\subsubsection{Biocular display below the users line of sight}

There is an additional variation of binocular displays, in which the two displays are positioned under the eye below the line of sight. The nearest analogy is reading glasses that are used by watching slightly downwards. The greatest advantage of this display type is that the user can easily glance to the environment without taking off the head-worn display. To test the use comfort with this type of glasses, we conducted a test series in which the users were required to complete a task in which they had to glance between the display and the surroundings. The test participants read a text from a paper and compared it to a text, which was shown in a virtual display.

Figure 1 shows the subjective symptom levels that are derived from SSQ questionnaire. Interestingly, the subjective symptom levels induced by the MicroOptical BV-3 display were not significantly different from the symptom levels induced by a CRT. This result clearly shows that a biocular display located below the line of sight is a comfortable alternative.
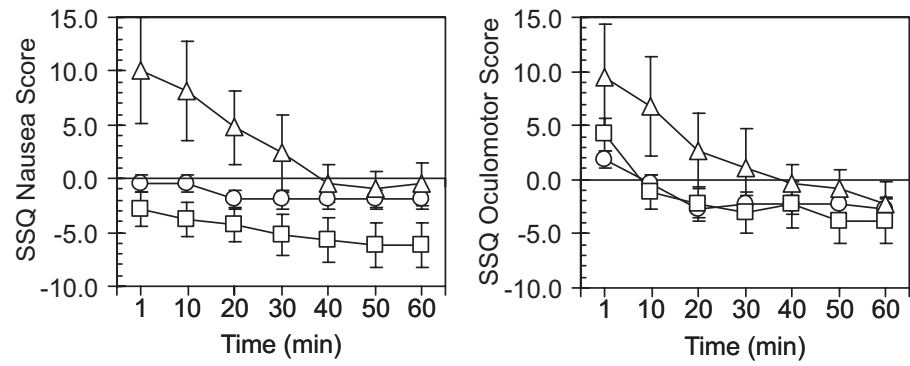

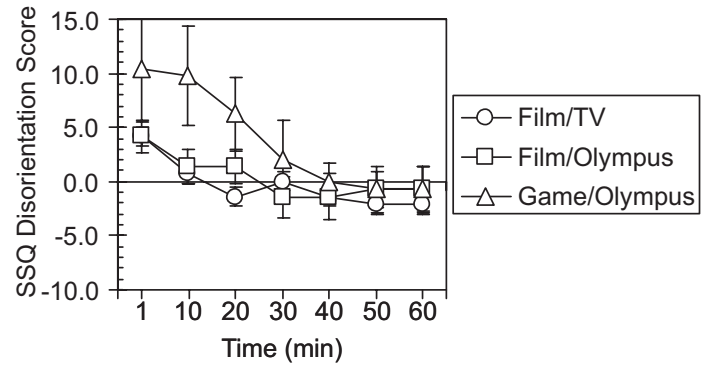

Figure 2. Symptom levels after task. 


\section{Conclusions}

There are several clear trends that can be seen in the results. First of all, displays do cause both subjective symptoms and objective physiological changes in the users. However, although some display types cause more adverse symptoms, other displays cause little or no symptoms at all. In some of our studies the users actually felt better after using the virtual display. Secondly, the experienced symptom levels vary according to the content, context and user group. For example, users who are used to playing computer games report significantly less adverse symptoms with virtual display gaming. Similarly, existing visual dysfunctions correlate to experienced symptoms.

Our experiments suggest that a two-eye display without stereoscopic graphics is the most comfortable alternative for users. Watching a movie with such a splay was a relaxing experience in which the symptom levels were minimized. Also, a biocular display located below the line of sight is an interesting alternative that should be further investigated. Our results thus show that a head-worn display as a mobile accessory is a viable alternative if proper attention is given to the ergonomics of the display.

\section{References}

[1] S.V.G. Cobb, S.C. Nichols, A.R. Ramsey, J.R. Wilson, Virtual Reality-Induced Symptoms and Effects (VRISE), Presence 8, 169-186 (1999).

[2] R.S. Kennedy, D.S. Lanham, J.M. Drexler, C.J. Massey, M.G. Lilienthal, A comparison of cybersickness incidences, symptom profiles, measurement techniques, and suggestions for further research, Presence 6, 638-644 (1997)

[3] S.M. Ebenholtz, Sources of asthenopia in navy flight simulators, New York, Institute for Vision Research SUNY, College of Optometry / Naval Training Systems Center: 11 (1988).

[4] M. Alpert, Machine chic. Scientific American, July 15, 2002.

[5] R.S. Kennedy, N.E. Lane, K.S. Berbaum, M.G. Lilienthal, Simulator sickness questionnaire: An enhanced method for quantifying simulator sickness., The International Journal of Aviation Psychology 3, 203-220 (1993).

[6] R.S. Kennedy \& K.M. Stanney, Postural instability induced by virtual reality exposure: Development of a certification protocol, International Journal of Human-Computer Interaction 8, 25-47 (1996).

[7] S.V.G. Cobb, Measurement of postural stability before and after immersion in a virtual environment, Applied Ergonomics 30, 47-57 (1999). 